\title{
Effect of Gestational Diabetes on Circulating Levels of Maternal and Neonatal Carnitine
}

Eleni Agakidou ${ }^{1 *}$, Elisavet Diamanti', loannis Papoulidis ${ }^{2}$, Evangelos Papakonstantinou ${ }^{3}$, loakeim Stergioudas ${ }^{4}$, Kosmas Sarafidis $^{1}$, Vasiliki Drossou ${ }^{1}$ and Athanasios Evangeliou

${ }^{1} 1^{\text {st }}$ Department of Neonatology of Aristotle University of Thessaloniki, Greece

${ }^{2}$ Eurogenetica Lab, Greece

${ }^{3}$ NeoLab, Athens, Greece

${ }^{4} 2^{\text {nd }}$ Department of Obstetrics and Gynecology of Aristotle University of Thessaloniki, Greece

${ }^{5} 4^{\text {th }}$ Department of Pediatrics, Aristotle University of Thessaloniki, Greece

\begin{abstract}
Objectives: Free carnitine is reported to decrease in normal pregnancies whereas data on the effect of gestational diabetes mellitus (GDM) on maternal and offspring carnitine status are lacking. This study aimed to assess changes in carnitine status in mothers with GDM and the offspring.

Subjects/methods: Prospective case-control study of 54 pairs of mothers - neonates (27 with GDM and 27 with normal pregnancies) and 26 non-pregnant controls. Serum glucose, lipids, and total fatty acids, blood $\beta$-hydroxybutyric acid, and whole blood carnitine were assessed in mothers before labor and neonates at birth. Carnitine was assessed in dried blood-spots on Guthrie paper using Tandem Mass Spectrometry.

Results: Compared to the controls, both groups of mothers had lower free carnitine and acylcarnitine and higher triglyceride and fatty acid levels. Compared to their respective maternal group, both groups of neonates had significantly higher free carnitine and acylcarnitine and lower triglyceride and fatty acid levels. Free carnitine and acylcarnitine, were comparable between the two maternal groups, whereas they were significantly higher in neonates of GDM mothers versus healthy neonates.

Conclusions: Well controlled GDM does not exacerbate changes in free carnitine, acylcarnitine, and fatty acid levels in pregnant women, albeit it is associated with increased carnitine in the newborn indicating enhanced fatty acid oxidation. The potential association of carnitine changes with macrosomia and long-term consequences in the offspring of GDM needs further investigation.
\end{abstract}

Keywords: Acylcarnitine; Dried blood spots; Fatty acids; Lipids; Pregnancy; Tandem mass spectrometry; Triglycerides

Abbreviations: AC: Acylcarnitine; FA: Fatty Acid; FC: Free Carnitine; GDM: Gestational Diabetes Mellitus; IDM: Infants of Diabetic Mothers

\section{Introduction}

Changes in intermediary metabolism occurring during pregnancy aim to meet the energy requirements of the pregnant women and ensure adequate nutrient supply to the fetus $[1,2]$. Carnitine ( $\beta$-hydroxy- $\gamma$ trimethylaminobutyric acid) is an essential cofactor that plays a central role in fatty acid (FA) metabolism by transferring the long-chain FAs across the inner mitochondrial membrane into the mitochondrial matrix, where they are catabolized via $\beta$-oxidation $[3,4]$. Impairment of the carnitine shuttle may adversely affect FA oxidation leading to serious complications, especially during metabolic derangements, including liver dysfunction, skeletal myopathy, cardiomyopathy, and hypoketotic hypoglycemia [5]. Several studies in normal pregnancies have documented low plasma free carnitine (FC) levels approaching those observed in organic acidurias which, however, were not associated with clinical manifestations of carnitine insufficiency [6-8].

Data on carnitine levels in women with gestational diabetes mellitus (GDM) are sparse. So far, there is only one published study on carnitine levels in pregnancies complicated with GDM, which reported increased plasma FC [9]. This finding is in disagreement with the decreased FC found in children and adolescents with type I diabetes [10]. Regarding the fetus/neonate, carnitine has been reported to be increased in neonates born to mothers with uncomplicated pregnancies $[6,11]$, whereas there are no published data on the effect of GDM on the neonatal carnitine levels. The primary aim of this study was to assess the effect of GDM on maternal and offspring's carnitine levels. In addition, we investigated the possible association between circulating carnitine levels and FA changes in the mothers and their neonates.

\section{Subjects and Methods}

This is a prospective case-control study. Participants were recruited among pregnant women who attended the antenatal clinic of the $2^{\text {nd }}$ Department of Obstetrics and Gynecology of the Aristotle University of Thessaloniki, at the Hippokration General Hospital, during a twoyear period. Eligible for the study were all pregnant women with single pregnancies who had an abnormal glucose tolerance test at 26 28 weeks of gestation and no history of pre-gestational diabetes. An

*Corresponding author: Eleni Agakidou, 1st Department of Neonatology Aristotle University of Thessaloniki, Hippokration Hospital, 28 Glinou str, 54352 Thessaloniki, Greece, Tel: +30 2310 992991; Fax: +30 2310 992787; E-mail: eagaki@hotmail.com

Received January 11, 2013; Accepted February 16, 2013; Published February 20, 2013

Citation: Agakidou E, Diamanti E, Papoulidis I, Papakonstantinou E, Stergioudas I, et al. (2013) Effect of Gestational Diabetes on Circulating Levels of Maternal and Neonatal Carnitine. J Diabetes Metab 4: 250. doi:10.4172/2155-6156.1000250

Copyright: (c) 2013 Agakidou E, et al. This is an open-access article distributed under the terms of the Creative Commons Attribution License, which permits unrestricted use, distribution, and reproduction in any medium, provided the original author and source are credited. 
equal number of pregnant women with a normal glucose tolerance test and uneventful single pregnancies comprised the group of nondiabetic pregnant women. Exclusion criteria were hypertension or pre-eclampsia during the current pregnancy, chronic maternal health conditions, and perinatal problems that could possibly affect the neonatal carnitine levels, including delivery prior to the $36^{\text {th }}$ week of gestation, perinatal asphyxia, congenital anomalies, evidence of intrauterine or perinatal infections, and metabolic diseases of the newborn. The study was approved by the Scientific Committee of the Hospital and the Ethics Committee of the Medical Faculty, Aristotle University of Thessaloniki, and written informed consent was obtained from all mothers prior to recruitment.

All pregnant women were screened for gestational diabetes between the $26^{\text {th }}$ and $28^{\text {th }}$ week of gestation. The 2 -hour oral glucose tolerance test using a loading dose of $75 \mathrm{~g}$ of glucose was performed. The plasma glucose threshold values for the diagnosis of gestational diabetes were $95 \mathrm{mg} / \mathrm{dL}, 180 \mathrm{mg} / \mathrm{dL}$, and $155 \mathrm{mg} / \mathrm{dL}$ for the fasting, 1-hour, and 2-hour glucose values, respectively. In the case of abnormal or ambiguous oral glucose tolerance test results the test was repeated. The level of metabolic control was evaluated with regular blood glucose checks and blood HbAlc assessment according to the department policy. During the study period 27 pregnant women with GDM (GDM mothers) and 27 non-diabetic pregnant women (non-GDM mothers) matched for age, gestational age and the mode of delivery were included in the study. In 15 GDM mothers, glucose control was managed with diet whereas 12 of them required insulin treatment. Twenty-six healthy non-pregnant female volunteers, matched for age, served as controls.

Recorded data included maternal history of health problems and obstetric history, current pregnancy complications and perinatal history. Anthropometric measurements included maternal weight, weight gain during pregnancy, height, maternal triceps skinfold thickness, birth weight (BW), length, and neonatal triceps and quadriceps skinfold thickness. The maternal body mass index (BMI=weight $[\mathrm{kg}] /$ height $[\mathrm{m}]^{2}$ ) a few days before delivery and neonatal ponderal index $\left(\mathrm{PI}=\left[\mathrm{BW}[\mathrm{g}] \times 100 /\right.\right.$ length $\left.[\mathrm{cm}]^{3}\right)$ at birth were calculated.

\section{Laboratory measurements}

Fasting blood samples were obtained from all pregnant women about 1 week prior to the expected delivery date and from the non-pregnant controls. Venous blood samples from the neonates were obtained within 1 hour after birth, before the first feeding. All measurements were performed in venous blood obtained by a single bleeding. Biochemical tests included measurement of blood $\beta$-hydroxybutyrate and serum glucose, triglycerides, total cholesterol, HDL- and LDL- cholesterol, and FAs. The serum triglycerides, total cholesterol, HDL- and LDL- cholesterol were assayed using the OLYMPUS AU2700 biochemical analyzer (Olympus, Hamburg, Germany). Measurement of $\beta$-hydroxybutyrate was performed in venous blood using the MEDISENSE ${ }^{\circ}$ OPTIMUM BLOOD GLUCOSE AND KETONE SENSOR (Abbott Laboratories, Hellas, S.A., Athens, Greece). The assay range is $0.0-6.0 \mathrm{mmol} / \mathrm{L}$. A previous study reported that the intra-assay coefficients of variation tested on three different levels of $\beta$-hydroxybutyrate (low at the mean of 0.43 , moderate at 1.08 , and high at $3.55 \mathrm{mmol} / \mathrm{L}$ ) were $10.5,5.5$, and $3.2 \%$, respectively [12].

Serum samples were stored at $-80^{\circ} \mathrm{C}$ until measurement of serum FAs. Serum FAs were measured according to the method described by Lagerstedt et al. [13] by using Gas Chromatography/Mass Spectrometry. The analyser used in our study was the AGILENT 7890A GAS CHROMATOGRAPHY (Agilent Technologies, Inc., Agilent
Technologies, Inc. 2850 Centerville Road Wilmington, DE 19808-1610 USA) equipped with an AGILENT 5975C EI MSD DETECTOR. In addition, we used two modifications of the method; first, separation was performed on a HP-5MS column $(30 \mathrm{~m} \times 250 \mu \mathrm{m} \times 0.25 \mu \mathrm{m})$ and second, the initial oven temperature was $70^{\circ} \mathrm{C}$, hold for $4 \mathrm{~min}$, and then temperature increased at a rate of $14^{\circ} \mathrm{C} / \mathrm{min}$ to $150^{\circ} \mathrm{C}$ and then with $4^{\circ} \mathrm{C} / \mathrm{min}$ to $210^{\circ} \mathrm{C}$.

For measurement of FC and AC fractions, $50 \mu \mathrm{L}$ of venous blood were carefully placed on filter paper (Guthrie cards) within the circle and were left to dry at ambient temperature for 24-48 hours before being sent to the laboratory. Measurements were performed using double quadruple mass spectrometer API2000 (Applied Biosystems, Foster City, CA 94404, USA) through an HPLC system (PE200, Perkin Elmer, Southfield, MI 48075, USA). Extraction was performed using methanol containing carnitine and acylcarnitine (AC) stable isotopelabelled internal standards and derivatization using $60 \mathrm{uL}$ butanol$\mathrm{HCl}$. The scientists who performed the measurements were unaware of the group assignment.

\section{Statistical analysis}

Carnitine and FA values were normally distributed in all groups (Kolmogorov-Smirnov test), whereas the gestational age and certain maternal anthropometric and biochemical parameters followed a non-Gaussian distribution. Numerical data were expressed as either means and standard deviations or medians with ranges, as appropriate. Differences were assessed using the unpaired $t$-test or Mann-Whitney test and the parametric ANOVA with the Dunnett T3 test for multiple comparisons or the Kruskal-Wallis non-parametric ANOVA with Dunn's test for multiple comparisons, as appropriate. The Fisher exact test was used to compare categorical variables. The Pearson or the Spearman correlation coefficients were used to assess bivariate correlations. Based on FC and AC values published by Schulpis et al. [11], who used the same technology to measure FC and AC in dried blood spots in healthy pregnant women, we calculated that in order to detect a difference in mean FC and AC values of $20 \%$ between normal and diabetic pregnancies, with an estimated standard deviation of FC and AC $4 \mu \mathrm{mol} / \mathrm{L}$ and $3 \mu \mathrm{mol} / \mathrm{L}$, respectively, a desired power of $80 \%$, and a 2 -tailed significance criterion of $0.05,25$ and 20 women in each group for the FC and AC, respectively, should be recruited. The calculation for sample size was performed using the on line calculator created by Eng that was accessed at http://www.rad.jhmi.edu/jeng/ javarad/samplesize/ [14]. Statistical analysis was performed by using the Statistical Software Package SPSS 16.0 (SPSS Inc., Chicago, IL) and the GraphPad InStat (version 3.05 GraphPad Software, San Diego, CA).

\section{Results}

\section{Clinical characteristics}

Among the anthropometric measurements only the BMI differed significantly between the two groups of pregnant women (Table 1). Compared to the controls, both groups of pregnant women had significantly higher BMI and triceps skinfold thickness (Table 1). No pregnant woman had any clinical signs of carnitine insufficiency. The neonates of non-GDM mothers, thereafter referred to as healthy neonates, did not differ significantly from the infants of diabetic mothers (IDM) regarding the perinatal data and anthropometric measurements (Table 2). Only two (8\%) IDM were macrosomic (birth weight higher than the $97^{\text {th }}$ centile for gestational age). However, 5 (18.5\%) IDM versus 1 (3.6\%) healthy neonate had birth weights higher than the $90^{\text {th }}$ centile for gestational age. 
Citation: Agakidou E, Diamanti E, Papoulidis I, Papakonstantinou E, Stergioudas I, et al. (2013) Effect of Gestational Diabetes on Circulating Levels of Maternal and Neonatal Carnitine. J Diabetes Metab 4: 250. doi:10.4172/2155-6156.1000250

Page 3 of 6

\begin{tabular}{|c|c|c|c|c|}
\hline & Controls & Non-GDM mothers & GDM mothers & $P^{a, b, c}$ \\
\hline Number & 26 & 27 & 27 & \\
\hline Age (years, $x \pm S D$ ) & $32.1 \pm 4.9$ & $30.4 \pm 5.6$ & $31.3 \pm 4.9$ & $N . S^{a}$ \\
\hline Weight gain in pregnancy $(\mathrm{kg}, \mathrm{x} \pm \mathrm{SD})$ & & $13.2 \pm 5.6$ & $12.3 \pm 4.3$ & $N . S .^{c}$ \\
\hline $\mathrm{BMI}\left(\mathrm{kg} / \mathrm{m}^{2}, \mathrm{x} \pm \mathrm{SD}\right)$ & $22.1 \pm 3.1^{* \dagger}$ & $27.9 \pm 4.8^{\ddagger}$ & $32.3 \pm 5.8$ & $<0.001^{a}$ \\
\hline TSFT (mm, $x \pm S D)$ & $19.0 \pm 9.6^{* \dagger}$ & $25.7 \pm 9.9$ & $32.0 \pm 9.8$ & $<0.001^{a}$ \\
\hline Blood glucose (mmol/L, $x \pm S D)$ & $5.8 \pm 0.6^{* \dagger}$ & $4.9 \pm 1.1$ & $4.9 \pm 0.9$ & $<0.001^{a}$ \\
\hline Blood $\beta$-hydroxy-butyrate (mmol/L, median [min - max]) & $0.3(0.1-0.3)$ & $0.2(0.1-1.0)$ & $0.2(0.1-0.8)$ & $N . S^{b}$ \\
\hline Triglycerides (mmol/L, $\mathrm{x} \pm \mathrm{SD}$ ) & $0.83 \pm 0.5^{* \dagger}$ & $3.00 \pm 1.1$ & $3.29 \pm 0.9$ & $<0.001^{a}$ \\
\hline Cholesterol (mmol/L, $\mathrm{x} \pm \mathrm{SD})$ & $5.33 \pm 1.1^{* \dagger}$ & $7.05 \pm 1.20$ & $6.94 \pm 1.23$ & $<0.001^{a}$ \\
\hline HDL cholesterol (mmol/L, $x \pm S D)$ & $1.65 \pm 0.25$ & $1.84 \pm 0.48$ & $1.67 \pm 0.41$ & N.S. ${ }^{a}$ \\
\hline LDL cholesterol (mmol/L, $x \pm S D)$ & $3.30 \pm 0.87$ & $3.60 \pm 1.32$ & $3.62 \pm 0.96$ & $N . S^{a}$ \\
\hline
\end{tabular}

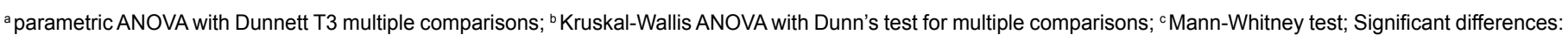
controls vs. non-GDM mothers; ${ }^{\dagger}$ control vs. GDM mothers; ${ }^{\ddagger}$ non-GDM vs. GDM mothers; NS: Not Significant; GDM: Gestational Diabetes Mellitus; TSFT: Triceps Skinfold Thickness

Table 1: Clinical and biochemical data of gestational diabetic versus non-diabetic, and non-pregnant (control) women.

\begin{tabular}{|c|c|c|c|c|}
\hline & Healthy neonates & IDM & $P a, b, c$ & OR $(95 \% \mathrm{Cl})$ \\
\hline Number & 27 & 27 & & \\
\hline GA (weeks, median [min - max]) & $38(36-40)$ & $38(36-39)$ & $N . S^{b}$ & N.A. $(0.02,0.93)$ \\
\hline$B W(g, x \pm S D)$ & $3143 \pm 317$ & $3176 \pm 429$ & $N . S^{a}$ & N.A. $(-242,162$ \\
\hline Cesarean delivery [n (\%)] & $12(44)$ & $14(52)$ & $N . S^{c}$ & $1.35(0.46,3.93)$ \\
\hline Male sex [n (\%)] & $15(56)$ & $16(59)$ & $N . S^{c}$ & $0.86(0.29,2.53)$ \\
\hline $\mathrm{PI}\left(\mathrm{g} / \mathrm{cm}^{3}, \mathrm{x} \pm \mathrm{SD}\right)$ & $2.45 \pm 0.26$ & $2.56 \pm 0.31$ & $N . S^{a}$ & N.A. $(-0.25,0.02)$ \\
\hline Triceps skinfold thickness $(\mathrm{mm}, \mathrm{x} \pm \mathrm{SD})$ & $4.6 \pm 0.89$ & $4.6 \pm 0.91$ & N.S. ${ }^{a}$ & N.A. $(-0.49,0.54)$ \\
\hline Quardiceps skinfold thickness (mm, $x \pm S D$ ) & $6.2 \pm 1.2$ & $6.8 \pm 2.2$ & $N . S^{a}$ & N.A. $(-1.61,0.35)$ \\
\hline Blood glucose $(\mathrm{mmol} / \mathrm{L}, \mathrm{x} \pm \mathrm{SD})$ & $4.2 \pm 1.1$ & $3.1 \pm 1.0$ & $.001^{a}$ & N.A. $(0.47,1.71)$ \\
\hline Blood $\beta$-hydroxy-butyrate (mmol/L, median [min - max]) & $0.2(0.1-0.4)$ & $0.2(0.1-0.3)$ & $N . S^{b}$ & N.A. \\
\hline Triglycerides (mmol/L, median [min - max]) & $0.44(0.25-2.21)$ & $0.60(0.27-2.02)$ & $0.007^{b}$ & N.A. \\
\hline Cholesterol $(\mathrm{mmol} / \mathrm{L}, \mathrm{x} \pm \mathrm{SD})$ & $1.77 \pm 0.39$ & $1.89 \pm 0.79$ & N.S. ${ }^{a}$ & N.A. $(-0.48,0.23)$ \\
\hline HDL cholesterol (mmol/L, $x \pm S D)$ & $0.94 \pm 0.24$ & $0.85 \pm 0.30$ & $N . S^{a}$ & N.A. $(-0.07,0.25)$ \\
\hline LDL cholesterol (mmol/L, $x \pm S D)$ & $0.65 \pm 0.19$ & $0.81 \pm 0.50$ & $N . S^{a}$ & N.A. $(-0.39,0.06)$ \\
\hline
\end{tabular}

at-test; b Mann-Whitney test; ' Fisher exact test; NS: Not Significant; IDM: Infants of Diabetic Mothers; NA: Not Applicable

Table 2: Clinical and biochemical data in neonates of gestational diabetic versus non-diabetic mothers.

\section{Biochemical parameters}

Comparison between the non-GDM and GDM mothers showed comparable levels of serum glucose, $\beta$-hydroxyburytic acid, and lipids (Table 1). In comparison with the controls, both groups of pregnant women had significantly lower blood glucose and higher serum triglycerides and cholesterol (Table 1). Comparison between the two groups of neonates showed that the IDM had significantly lower blood glucose, although only 2 of them developed hypoglycemia (blood glucose lower than $2 \mathrm{mmol} / \mathrm{L}$ ). In addition, the IDM had significantly higher serum triglyceride levels (Table 2).

\section{Blood carnitine}

Results regarding the levels of total carnitine, FC, and AC fractions in blood and comparisons among the groups of mothers and neonates are summarized in table 3 (Supplementary file-Figure 1). Values of AC are presented as sum of total $\mathrm{AC}$, as well as major AC fractions, namely short-chain AC (C2- C5), medium-chain AC (C6 - C12), and longchain AC (C14 - C18). The two groups of mothers had comparable FC, AC, and AC fraction levels. In comparison to the controls, both maternal groups had significantly lower FC, whereas the non-GDM but not the GDM mother had significantly lower AC. In all neonates carnitine fraction levels were significantly higher than in their respective mothers, except for the medium-chain AC. Comparison between healthy neonates and IDM showed significantly higher FC,
$\mathrm{AC}$, and short-chain $\mathrm{AC}$ in the IDM, whereas the difference in longchain AC was of borderline significance (Table 3).

\section{Serum fatty acid levels}

The two groups of mothers had total FA and FA fractions significantly higher than the control levels. The neonates had total FA, long-chain (C13-C18) FA, and very long-chain (C20-C22) FA lower than the respective maternal levels. The FAs did not differ either between the two maternal groups or between the two neonatal groups (Table 4).

\section{Correlations}

The maternal FC, AC, and AC fractions were significantly positively correlated with the respective neonatal carnitine levels (Table 5). The maternal total FAs and FA fractions were not correlated with the respective neonatal FA levels (Table 5). Among the lipids, the triglycerides were significantly positively correlated the AC levels in the mothers $(\mathrm{r}=0.279, p=0.043)$ and the neonates $(\mathrm{r}=0.373, p=0.006)$.

\section{Discussion}

Our results showed that GDM was associated with increased FC, $\mathrm{AC}$, and short-chain $\mathrm{AC}$ in the neonate whereas it did not further compromise the maternal carnitine concentrations. No mother or neonate had clinical manifestations of carnitine insufficiency.

The low circulating FC levels in normal pregnancies documented 


\begin{tabular}{|c|c|c|c|c|c|}
\hline & Controls $(n=26)$ & Non-GDM mothers $(n=27)$ & GDM mothers $(n=27)$ & Healhy neonates $(n=27)$ & IDM $(n=27)$ \\
\hline TC & $53.3 \pm 9.0$ & $24.5 \pm 4.4^{*}$ & $27.5 \pm 7.8^{*}$ & $40.8 \pm 8.9^{\ddagger}$ & $55.0 \pm 13.0$ \\
\hline FC & $42.3 \pm 7.9$ & $15.6 \pm 3.3^{*}$ & $18.8 \pm 6.6^{*}$ & $24.8 \pm 6.2^{\ddagger}$ & $33.3 \pm 8.1^{\dagger}$ \\
\hline tAC & $11.0 \pm 2.2$ & $8.8 \pm 1.9^{*}$ & $10.7 \pm 4.6$ & $16.2 \pm 4.4^{\ddagger}$ & $21.7 \pm 5.9^{\dagger}$ \\
\hline SCAC & $8.6 \pm 1.4$ & $7.0 \pm 1.8^{*}$ & $8.9 \pm 4.2$ & $12.6 \pm 3.8^{\ddagger}$ & $17.7 \pm 5.1^{\dagger}$ \\
\hline MCAC & $0.60 \pm 0.23$ & $0.34 \pm 0.06^{*}$ & $0.36 \pm 0.09^{*}$ & $0.37 \pm 0.15$ & $0.36 \pm 0.11^{\dagger}$ \\
\hline LCAC & $1.8 \pm 0.4$ & $1.5 \pm 0.3^{*}$ & $1.5 \pm 0.5$ & $3.0 \pm 0.8$ & $3.8 \pm 1.2^{\dagger}$ \\
\hline
\end{tabular}

Values are expressed as means and standard deviations; parametric ANOVA with Dunnett T3 test for multiple comparisons; significant differences: *mothers vs. controls; ${ }^{*}$ IDM vs. controls; ${ }^{\ddagger}$ healthy neonates vs. IDM; The differences of all carnitine fractions (except for the MCAC) between the each maternal group and the respective neonatal group as well as between controls and healthy neonates were significant at $p<0.001$; no difference between the two maternal groups was significant; IDM: Infants of Diabetic Mothers; TC: Total Carnitine; FC: Free Carnitine; tAC: Total Acyl-Carnitine; SCAC: Short-Chain AC; MCAC: Medium-Chain AC; LCAC: Long-Chain AC

Table 3: Whole blood levels ( $\mu \mathrm{mol} / \mathrm{L}$ ) of free carnitine, total acyl-carnitine, and acyl-carnitine fractions.

\begin{tabular}{|c|c|c|c|c|c|}
\hline & Controls $(n=26)$ & Non-GDM mothers $(n=27)$ & GDM mothers $(n=27)$ & Healthy neonates $(n=27)$ & IDM (n=27) \\
\hline Total FAs & $7054 \pm 2231$ & $9735 \pm 2849^{* \dagger}$ & $10182 \pm 2427^{* \dagger}$ & $3524 \pm 722^{\ddagger}$ & $3996 \pm 2000^{\ddagger}$ \\
\hline $\mathrm{C} 8-\mathrm{C} 12$ & $27.3 \pm 10.3$ & $45.2 \pm 18.9^{*}$ & $38.5 \pm 14.4^{*}$ & $43.1 \pm 14.9^{\ddagger}$ & $42.4 \pm 14.7^{\ddagger}$ \\
\hline C13-C18 & $6223 \pm 1984$ & $8589 \pm 2642^{* \dagger}$ & $9044 \pm 2196^{* \dagger}$ & $2791 \pm 613^{\ddagger}$ & $3163 \pm 1632^{\ddagger}$ \\
\hline $\mathrm{C} 20-\mathrm{C} 22$ & $794 \pm 243$ & $1100 \pm 272^{* \dagger}$ & $1106 \pm 281^{*+}$ & $690 \pm 136$ & $791 \pm 378$ \\
\hline
\end{tabular}

Values are expressed as means and standard deviations; parametric ANOVA with Dunnett T3 test for multiple comparisons; significant differences: * mothers vs. controls; ${ }^{\dagger}$ mothers vs. respective neonatal group; ${ }^{\ddagger}$ neonates vs. controls; no difference either between the two groups of mothers or between the two groups of neonates was significant; IDM: Infants of Diabetic Mothers; FAs: Fatty Acids; C8-C12: Medium-Chain FAs; C13-C18: Long-Chain FAs; C20-C22: Very Long-Chain FAs

Table 4: Serum levels ( $\mu \mathrm{mol} / \mathrm{L})$ of fatty acids.

\begin{tabular}{|c|c|c|c|c|c|c|}
\hline & Neonatal FC r $(P)$ & Neonatal AC r $(P)$ & Neonatal SCAC r $(P)$ & Neonatal MCAC r $(P)$ & Neonatal LCAC r $(P)$ & Neonatal FAs $\mathrm{r}(P)$ \\
\hline Maternal FC & $0.55(<0.0001)$ & & & & & \\
\hline Maternal AC & & $0.43(<0.0001)$ & & & & \\
\hline Maternal SCAC & & & $0.47(<0.0001)$ & & & \\
\hline Maternal MCAC & & & & $0.07(0.603)$ & & \\
\hline Maternal LCAC & & & & & $0.29(0.030)$ & \\
\hline Maternal FAs & & & & & & $-0.19(0.890)$ \\
\hline
\end{tabular}

r: Pearson Correlation Coefficient; FC: Free Carnitine; AC: Acyl-Carnitine; SCAC: Short-Chain AC; MCAC: Medium-Chain AC; LCAC: Long-Chain AC; FAs: Fatty Acids

Table 5: Correlations between maternal and neonatal carnitine levels and fatty acids.

in the present study, as in several previous reports [6-8], have been attributed to transplacental transfer of FC to the fetus, which is not capable of producing carnitine. This is attributed to low activity of the enzyme $\gamma$-butyrobetaine hydroxylase being about $12 \%$ of adult values at birth $[15,16]$. Although no clinical evidence of carnitine insufficiency in pregnancy has been observed, the possible involvement of the reduced FC in the metabolic changes observed on normal pregnancy cannot be disputed. The increased serum triglycerides, cholesterol and FAs in late pregnancy have been attributed to the effect of the hormonal alterations occurring in pregnancy [17]. However, the low FC along with low AC suggests attenuated lipid oxidation. Consequently, the low FC observed in normal pregnancy can potentially contribute to accumulation of triglycerides and FAs by inhibiting $\beta$-oxidation. Moreover, our findings support the view that the excess of lipids is not used by the mother but it is rather transferred to the fetus. The transport of FAs to the fetus is facilitated by the presence of lipoprotein receptors together with lipoprotein lipase, phospholipase A2, and intracellular lipases in the placenta, which allow the free FAs to be released from maternal lipoproteins and triglycerides, transferred through the placenta and utilized by the fetus $[18,19]$. This process is very important for normal fetal development, which requires the availability of essential FAs and long-chain polyunsaturated FAs for growth and development [20].

The regulation of metabolic pathways may be substantially different in GDM compared to normal pregnancy. Data on the impact of diabetes on carnitine levels are controversial. Studies in juvenile type 1-diabetes showed decreased FC and increased AC [10] whereas other studies concluded that carnitine deficiency is not common in juvenile diabetes
[21]. Data regarding the carnitine status in GDM are rare. In our study, neither the FC nor the total AC and AC fractions differed between diabetic and normal pregnant women. This finding could be attributed to the good glycemic control of maternal diabetes, which provided a metabolic balance similar to that of normal pregnancy. This assumption is supported by the lack of fasting ketosis in our GDM population and it is in line with previous studies that reported comparable fasting ketonemia in normal pregnancies and well-controlled GDM [22]. Similarly, studies in diabetic patients showed that the FC levels are not decreased in the absence of diabetes-associated complications [23] or ketosis [24]. In contrast to our results, the only previous study dealing with carnitine levels in diabetic pregnancies reported increased FC and decreased AC in pregnancies complicated with GDM compared to normal pregnancies [9]. We can not find a plausible explanation for the increased FC found in the previous study. The different assay (radioisotopic) used by Pappa et al. [9] and measurements in plasma instead of whole blood may have contributed to the different results.

Regarding the effect of GDM on maternal serum lipids, the concentrations of triglycerides and FAs did not differ between the normal and GDM pregnant women. These results contrast previous reports of accentuated dyslipidemia in women with GDM [25]. The only plausible explanation for this disparity could be the good glycemic control of diabetes in our study population. In fact, previous studies in well-controlled GDM reported that the whole body net carbohydrate and FA utilization did not differ significantly between the GDM patients and control subjects [26]. Other authors also reported comparable levels of maternal FAs between normal and diabetic pregnancies [27]. 
These data suggest that well controlled GDM does not exacerbate the effect of pregnancy on FA metabolism.

Sufficient carnitine supply is very important for the fetus/neonate to stimulate FA oxidation and improve glucose homeostasis in order to meet the high energy requirements during delivery and early postnatal life [28]. In agreement with previous reports in cord blood, the levels of FC and AC were increased in the healthy neonates immediately after birth compared to the respective maternal values $[6,7,11]$. This finding is suggestive of an active, carrier-mediated transplacental transfer of FC to the fetus. The active transport of carnitine through the placenta is supported by experimental studies documenting the presence of the carnitine transporter organic cation transporter novel type II (OCTN2) in the placenta [29]. Levels of triglycerides and FAs were decreased in the neonates compared to the respective maternal levels. This finding could be due to either decreased transplacental transfer or increased utilization of FAs by the fetus/neonate. The notion of decreased transplacental transfer contrasts the high maternal FA levels as well as the findings of previous studies demonstrating the presence of lipoprotein receptors and enzymes in the placenta, which facilitate the free FAs transplacental transfer to the fetus $[18,19]$. On the other hand, the elevated FC and ACs found in the neonates support the notion of enhanced FA utilization by the fetus. This is in agreement with experimental studies showing high activity of the enzymes involved in FA oxidation in the human placenta [30] and the fetus [31].

So far there is no published data regarding the effect of GDM on the fetal/neonatal levels of carnitine. In our study, the IDM had significantly higher FC and AC levels than the healthy neonates. These findings are suggestive of increased $\beta$-oxidation in the IDM that is facilitated by the potentially increased FC supply via the placenta. The increased triglyceride levels in the IDM are also compatible with enhanced $\beta$-oxidation. It seems therefore that the GDM affects the carnitine-associated metabolic pathways of the fetus/neonate even in pregnancies with good glycemic control. Future studies may delineate the role of the carnitine changes and increased $\beta$-oxidation on the fetal metabolism and the short- and long-term consequences on the offspring of pregnancies complicated by GDM.

The strength of our study is that it is the first to report that the GDM affects the carnitine status in the offspring and that the changes are suggestive of increased $\beta$-oxidation. A weakness of our study is the fact that, due to limitations on the quantities of blood allowed to be withdrawn from neonates, we did not measure the neonatal serum insulin, which could add useful information regarding the glucose metabolism in the IDM.

In conclusion, our results indicate that gestational diabetes is associated with carnitine shuttle alterations in the fetus/neonate, which are compatible with enhanced FA oxidation, while it does not exacerbate the changes in carnitine-shuttle system occurring in the normal pregnant women. The potential association of the carnitine changes found in the offspring of GDM pregnancies with fetal programming during early pregnancy, i.e. before diagnosis of GDM and treatment implementation, and consequently, with the short- and long-term effects of GDM on the offspring needs further investigation.

\section{References}

1. Ghio A, Bertolotto A, Resi V, Volpe L, Di Cianni G (2011) Triglyceride metabolism in pregnancy. Adv Clin Chem 55: 133-153.

2. Forsum E, Löf M (2007) Energy metabolism during human pregnancy. Annu Rev Nutr 27: 277-292.
3. Rebouche CJ (2004) Kinetics, pharmacokinetics, and regulation of L-carnitine and acetyl-L-carnitine metabolism. Ann N Y Acad Sci 1033: 30-41.

4. Wutzke KD, Lorenz $\mathrm{H}$ (2004) The effect of I-carnitine on fat oxidation, protein turnover, and body composition in slightly overweight subjects. Metabolism 53 1002-1006.

5. Rinaldo P, Raymond K, al-Odaib A, Bennett MJ (1998) Clinical and biochemical features of fatty acid oxidation disorders. Curr Opin Pediatr 10: 615-621.

6. Talián GC, Komlósi K, Decsi T, Koletzko B, Melegh B (2007) Determination of carnitine ester patterns during the second half of pregnancy, at delivery, and in neonatal cord blood by tandem mass spectrometry: complex and dynamic involvement of carnitine in the intermediary metabolism. Pediatr Res 62: 88-92.

7. Koumantakis E, Sifakis S, Koumantaki Y, Hassan E, Matalliotakis I, et al (2001) Plasma carnitine levels of pregnant adolescents in labor. J Pediatr Adolesc Gynecol 14: 65-69.

8. Schoderbeck M, Auer B, Legenstein E, Genger H, Sevelda P, et al. (1995) Pregnancy-related changes of carnitine and acylcarnitine concentrations of plasma and erythrocytes. J Perinat Med 23: 477-485.

9. Pappa KI, Anagnou NP, Salamalekis E, Bikouvarakis S, Maropoulos G, et al. (2005) Gestational diabetes exhibits lack of carnitine deficiency despite relatively low carnitine levels and alterations in ketogenesis. J Matern Fetal Neonatal Med 17: 63-68.

10. Evangeliou A, Gourgiotis D, Karagianni C, Markouri M, Anogianaki N, et al (2010) Carnitine status and lactate increase in patients with type I juvenile diabetes. Minerva Pediatr 62: 551-557.

11. Schulpis KH, Papakonstantinou ED, Vlachos GD, Vlachos DG, Antsaklis A, et al. (2008) The effect of the mode of delivery on the maternal-neonatal carnitine blood levels and antioxidant status. Clin Chem Lab Med 46: 680-686.

12. Guerci B, Benichou M, Floriot M, Bohme P, Fougnot S, et al. (2003) Accuracy of an electrochemical sensor for measuring capillary blood ketones by fingerstick samples during metabolic deterioration after continuous subcutaneous insulin infusion interruption in type 1 diabetic patients. Diabetes Care 26: 1137-1141.

13. Lagerstedt SA, Hinrichs DR, Batt SM, Magera MJ, Rinaldo $P$, et al. (2001) Quantitative determination of plasma c8-c26 total fatty acids for the biochemical diagnosis of nutritional and metabolic disorders. Mol Genet Metab 73: 38-45.

14. Eng J (2003) Sample size estimation: how many individuals should be studied? Radiology 227: 309-313.

15. Winter SC, Linn LS, Helton E (1995) Plasma carnitine concentrations in pregnancy, cord blood, and neonates and children. Clin Chim Acta 243: 87-93.

16. Rebouche CJ, Engel AG (1980) Tissue distribution of carnitine biosynthetic enzymes in man. Biochim Biophys Acta 630: 22-29.

17. Pedersen SB, Kristensen K, Hermann PA, Katzenellenbogen JA, Richelsen B (2004) Estrogen controls lipolysis by up-regulating alpha2A-adrenergic receptors directly in human adipose tissue through the estrogen receptor alpha. Implications for the female fat distribution. J Clin Endocrinol Metab 89: 18691878.

18. Gil-Sánchez A, Koletzko B, Larqué E (2012) Current understanding of placental fatty acid transport. Curr Opin Clin Nutr Metab Care 15: 265-272.

19. Herrera E, Amusquivar E, López-Soldado I, Ortega H (2006) Maternal lipid metabolism and placental lipid transfer. Horm Res 65: 59-64.

20. Herrera $E$ (2002) Lipid metabolism in pregnancy and its consequences in the fetus and newborn. Endocrine 19: 43-55.

21. Jacobson JD, Midyett LK, Uttam Garg U, Sherman AK, Patel C (2011) Biochemical evidence for reduced carnitine palmitoyl transferase 1 (CPT-1) activity in type 1 diabetes mellitus. J Diabetes Metab 2: 144.

22. Gin H, Vambergue A, Vasseur C, Rigalleau V, Dufour P, et al. (2006) Blood ketone monitoring: a comparison between gestational diabetes and nondiabetic pregnant women. Diabetes Metab 32: 592-597.

23. Poorabbas A, Fallah F, Bagdadchi J, Mahdavi R, Aliasgarzadeh A, et al. (2007) Determination of free L-carnitine levels in type II diabetic women with and without complications. Eur J Clin Nutr 61: 892-895.

24. Soltész G, Melegh B, Sándor A (1983) The relationship between carnitine and ketone body levels in diabetic children. Acta Paediatr Scand 72: 511-515.

25. Sánchez-Vera I, Bonet B, Viana M, Quintanar A, Martín MD, et al. (2007) 
Citation: Agakidou E, Diamanti E, Papoulidis I, Papakonstantinou E, Stergioudas I, et al. (2013) Effect of Gestational Diabetes on Circulating Levels of Maternal and Neonatal Carnitine. J Diabetes Metab 4: 250. doi:10.4172/2155-6156.1000250

Page 6 of 6

Changes in plasma lipids and increased low-density lipoprotein susceptibility to oxidation in pregnancies complicated by gestational diabetes: consequences of obesity. Metabolism 56: 1527-1533.

26. Hsu HW, Butte NF, Wong WW, Moon JK, Ellis KJ, et al. (1997) Oxidative metabolism in insulin-treated gestational diabetes mellitus. Am J Physiol 272 E1099-1107.

27. Ortega-Senovilla H, Alvino G, Taricco E, Cetin I, Herrera E (2009) Gestational diabetes mellitus upsets the proportion of fatty acids in umbilical arterial but not venous plasma. Diabetes Care 32: 120-122.

28. Ringseis R, Keller J, Eder K (2012) Role of carnitine in the regulation of glucose homeostasis and insulin sensitivity: evidence from in vivo and in vitro studies with carnitine supplementation and carnitine deficiency. Eur J Nutr 51: 1-18.

29. Grube M, Meyer Zu Schwabedissen H, Draber K, Präger D, Möritz KU, et al (2005) Expression, localization, and function of the carnitine transporter octn2 (slc22a5) in human placenta. Drug Metab Dispos 33: 31-37.

30. Oey NA, den Boer ME, Ruiter JP, Wanders RJ, Duran M, et al. (2003) High activity of fatty acid oxidation enzymes in human placenta: implications for fetalmaternal disease. J Inherit Metab Dis 26: 385-392.

31. Oey NA, den Boer ME, Wijburg FA, Vekemans M, Augé J, et al. (2005) Long chain fatty acid oxidation during early human development. Pediatr Res 57 755-759. 